\title{
Aporte de hojarasca en bosques del predelta del río Paraná (Argentina)
}

\section{Litterfall production of forests at the pre-Delta area of the Paraná River (Argentina)}

\author{
Pablo Gilberto Aceñolaza ${ }^{a, b *}$, Lisandra Pamela Zamboni ${ }^{\mathrm{b}}$, Juan Fernando Gallardo Lancho ${ }^{\mathrm{c}}$

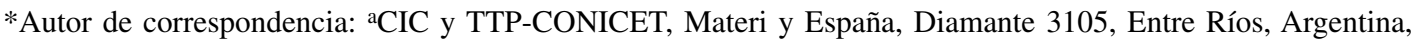 \\ tel/fax: 54343 4983086/87, acenolaza@gmail.com \\ bCEREGeo-FCyT, Universidad Autónoma de Entre Ríos, Argentina. \\ cC.S.I.C., IRNASA, Salamanca, España.
}

\begin{abstract}
SUMMARY
Litter production in four forests of the alluvial plain of the pre-Delta area in the Paraná River (Argentina) was studied during the period 1998-2002. Monospecific forests dominated by Salix humboldtiana (SSh) and Tessaria integrifolia (STi) presenting similar production $\left(6.8 \pm 1.2\right.$ and $6.5 \pm 1.3 \mathrm{Mg}$ dry matter $\mathrm{ha}^{-1} \mathrm{yr}^{-1}$, respectively), and pluri-specific forests of Albizia inundata (MAi) and Nectandra angustifolia $(\mathrm{MNa})$, presenting statistical differences in their average production. Plurispecific forests also showed significant differences between them in terms of production $\left(3.3 \pm 0.5\right.$ and $9.6 \pm 1.1 \mathrm{Mg}$ dry matter ha ${ }^{-1} \mathrm{yr}^{-1}$, respectively). The highest values of litter production were obtained during 1998 (year of the highest overflooding); afterwards, it started decreasing during the period studied. A seasonal pattern of litterfall was enhanced with the flood pulse (spring-summer, primarily from November to February), except in the Mai forest, where trees are deciduous (autumn leaf abscission). Regarding the composition of the litter, leaves are the most abundant fractions (reaching 69\% of the total in SSh forest), while reproductive structures account for less than $3 \%$ of the litter produced in all studied forests.
\end{abstract}

Key words: litter production, alluvial plain, overflooding pulses, forest biomass.

\section{RESUMEN}

Se estudió la caída de hojarasca en cuatro bosques representativos de la llanura aluvial del río Paraná, área predelta (Argentina), durante el período 1998-2002. Los bosques monoespecíficos dominados por Salix humboldtiana (SSh) y Tessaria integrifolia (STi) presentaron valores de producción aérea similares entre sí $\left(6,8 \pm 1,2\right.$ y $6,5 \pm 1,3 \mathrm{Mg}$ materia seca ha ${ }^{-1}$ año $^{-1}$, respectivamente) y se diferenciaron significativamente de los bosques mixtos de Albizia inundata (timbó blanco, MAi) y Nectandra angustifolia (laurel del río, $\mathrm{MNa})$. Los bosques mixtos también presentaron diferencias significativas entre sí en cuanto a dicho aporte $(3,3 \pm 0,5 \mathrm{y}$ 9,6 $\pm 1,1 \mathrm{Mg}$ materia seca ha ${ }^{-1} \mathrm{año}^{-1}$, respectivamente); los valores más altos de aporte de hojarasca se obtuvieron durante 1998 (año de fuerte crecida del río), decreciendo luego durante el tiempo estudiado. Se observó un patrón de aporte de hojarasca estacional, que se acentuó con la finalización del pulso de inundación (primavera-verano, principalmente de noviembre a febrero), salvo en el bosque MAi, que por su condición de caducifolio presentó una caída otoñal característica. En relación a la composición de la hojarasca, las hojas son las fracciones más representadas (alcanzando 69\% del total en el bosque SSh), mientras que las estructuras reproductivas suponen menos del $3 \%$ de la hojarasca producida en estos bosques.

Palabras clave: caída de hojarasca, llanura aluvial, pulso de inundación, biomasa forestal.

\section{INTRODUCCIÓN}

Gran parte de la materia orgánica del suelo (MOS) deriva de la humificación de los residuos orgánicos que llegan al suelo tras su abscisión: hojas, ramas, flores, frutos y demás estructuras vegetales (incluso árboles enteros) que conforman la hojarasca. Esta MOS, tras su mineralización, libera bioelementos que pueden ser reabsorbidos por las plantas, contribuyendo así al ciclo biogeoquímico. Esta liberación de nutrientes, por tanto, está determinada, en última instancia, por las características genéticas de las especies, la variación anual de la composición de la hojarasca, sus propiedades físico-químicas y por las con- diciones ambientales, determinando el ritmo de cesión de nutrientes en cada ecosistema forestal. En este contexto, el análisis del aporte de hojarasca al suelo constituye una forma apropiada de estimar la productividad forestal (Santa Regina et al. 1991, Haase 1999, Carnevale y Lewis 2001, Zamboni y Aceñolaza 2004, Aceñolaza et al. 2006).

La estacionalidad del componente foliar, evaluada a través de su productividad y descomposición (dada su elevada sensibilidad a la variación de las características mesológicas que condicionan la comunidad arbórea), justifica el empleo de la dinámica foliar como indicador ambiental. Las estimaciones de producción primaria, tanto neta como bruta, requieren de una inversión importante en tiempo 
y trabajo, resultando más práctico y sencillo estimar la caída de hojarasca (Monedero y González 1995). En este contexto, cabe mencionar que la producción de hojarasca cumple con importantes funciones en la productividad forestal (Bray y Gorham 1964) y, aunque no constituye una estimación completa de la producción de fitomasa, representa el componente de la vegetación de crecimiento más intenso (UNESCO-PNUMA-FAO 1980).

En las llanuras aluviales fluviales todos estos procesos están estrechamente asociados a las características hidrológicas (Brinson et al. 1980, Junk et al. 1989) o al pulso hidrosedimentológico (Neiff y Poi de Neiff 1990). Así, en los bosques fluviales, sujetos a inundaciones estacionales, la entrada y salida del agua les confiere dinámicas propias en lo referente a los ciclos biogeoquímicos presentando, por lo general, altas producciones. Además, si la temperatura ambiental es suficientemente alta se produce un mayor ritmo de descomposición de la materia orgánica acumulada sobre el suelo (horizonte $O$, o necromasa) que en otros sistemas netamente acuáticos o no inundables (Junk et al. 1989, Haase 1999).

La existencia de picos en los procesos ecológicos de estos bosques con inundaciones recurrentes (con especies tolerantes a la hidromorfía temporal) les confiere una actividad pulso-dependiente. Así, la caída de hojas suele estar relacionada con la fase de inundación, aunque pueda también existir desfoliación menos intensa a lo largo del año. La fructificación, la germinación y el crecimiento de semillas también suelen estar asociados a la fase de inundación (Godoy et al. 1999).

Sudamérica cuenta con alguna información sobre productividad de bosques fluviales, pero ya Proctor (1983, 1984) alertó sobre la falta de información para bosques estacionales del mismo subcontinente en su revisión sobre los procesos de aporte de hojarasca de bosques tropicales sujetos a inundación. Posteriormente, Haase (1999) indicó que esta situación no había cambiado significativamente.

En lo referente a la llanura aluvial del río Paraná (Argentina), área de estudio del presente trabajo, la información es más bien limitada y se reduce a los trabajos de Neiff y Poi de Neiff (1990) sobre bosques de aliso del río (Tessaria integrifolia Ruiz et Pav.; STi) en el Paraná Inferior; y de Zamboni y Aceñolaza (2004) y Aceñolaza et al. (2006), quienes analizaron los patrones y cadencia de aporte de hojarasca en bosques de STi, sauce criollo (Salix humboldtiana Willd.; SSh) y timbó blanco (Albizia inundata (Mart.) Barneby et Grimes; MAi) localizados en el predelta del río Paraná.

Según Zamboni y Aceñolaza (2004) en el área de estudio (predelta) se reconocen sistemas boscosos con características propias (en cuanto a composición específica, características estructurales, fenología, floración, fructificación, dispersión de semillas, producción de biomasa, caída del material vegetal, entre otros). Distinguieron sistemas de: a) bosques monoespecíficos, de SSh (generalmente monoespecífico de $S$. humboldtiana) y de STi (dominados por T. integrifolia), con escaso desarrollo de estratos herbáceo y arbustivo, que se ubican en bancos de arena del cauce (sometidos, por ello, a inundaciones estacionales de alta energía) y actuando como especies colonizadoras; y b) bosques "mixtos" de MAi (dominado por $A$. inundata) y laurel del río (dominado por Nectandra angustifolia (Schrad.) Nees et Mart.; MNa), los cuales poseen mayor riqueza específica, con estratos claramente desarrollados y existencia de lianas, y que se ubican en áreas más elevadas (albardones internos), con influencia más bien indirecta de las variaciones hidrológicas.

El aporte de hojarasca de la especie dominante puede constituir una variable importante que permite examinar las fenologías propias de estos tipos de bosques. En los bosques monoespecíficos el patrón de aporte de hojarasca de la especie dominante puede describir al patrón de aporte del bosque; mientras que en los bosques mixtos ya no es posible adjudicar un patrón de aporte en función de la especie dominante (Zamboni y Aceñolaza 2004).

Los objetivos del presente estudio son: a) conocer y comparar los aportes de hojarasca en bosques del predelta del río Paraná; b) analizar si existe relación entre el patrón de aporte de hojarasca del bosque y el régimen hidrológico; c) describir y comparar la contribución de las especies dominantes, tanto en bosques monoespecíficos como en mixtos.

\section{MÉTODOS}

Área de estudio. El estudio se llevó a cabo en el Parque Nacional Pre-Delta (PNPD; localización: $32^{\circ} 03^{\prime} 43^{\prime \prime}$ S y $60^{\circ} 38^{\prime} 39^{\prime \prime} \mathrm{O}$ ), ubicado a $5 \mathrm{~km}$ de la ciudad de Diamante (Entre Ríos, Argentina). El PNPD (figura 1) es un área protegida desde 1992 bajo jurisdicción de la Administración de Parques Nacionales (APN); esta situación ha permitido la conservación de paisajes no alterados por el ganado (uno de los principales factores de deterioro de la región).

El PNPD tiene una superficie de $24,6 \mathrm{~km}^{2}$, mayormente representado por cuerpos de agua, sectores isleños y barrancas. El clima es templado-cálido y húmedo. La temperatura media anual es alrededor de $19^{\circ} \mathrm{C}$, mientras que la pluviosidad media anual es de $1.126 \mathrm{~mm}$ año ${ }^{-1}$, concentrándose las lluvias en primavera (media de $348 \mathrm{~mm}$ ) y verano (media de 425 mm; Rojas y Saluso 1987). Hay que indicar que, en los últimos años, las lluvias se han registrado con mayor frecuencia hacia el otoño (incluyendo también el mes de abril), lo cual acentúa localmente el nivel de inundación y, sobre todo, la disponibilidad de agua edáfica.

El régimen hidrológico del sistema del Paraná presenta un pulso anual de inundación (unimodal), generalmente en los meses de otoño (abril a junio, esto es, cuando se reducen las precipitaciones), seguido de un período de estiaje en verano. En todo caso, durante el verano se presenta un período de déficit hídrico edáfico (Neiff y Poi de 


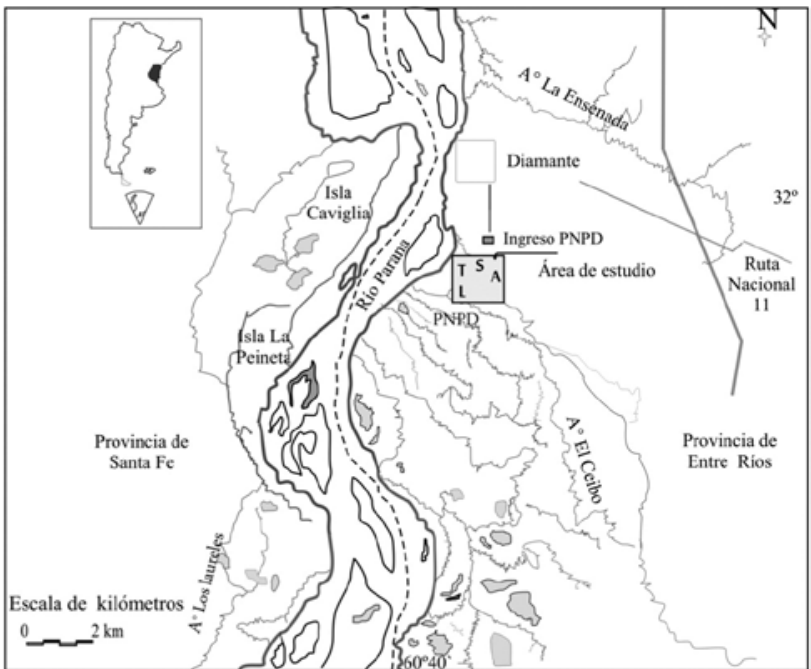

Figura 1. Ubicación geográfica del área de estudio $\left(32^{\circ} 03^{\prime}\right.$ $43^{\prime \prime} \mathrm{S}$ y $\left.60^{\circ} 38^{\prime} 39^{\prime \prime} \mathrm{O}\right)$. Se destaca la cercanía del área de estudio al río Paraná (Argentina). Se ha delimitado el área de estudio y la ubicación de los bosques estudiados. T: Tessaria integrifolia; A: Albizia inundata; L: Nectandra angustifolia y S: Salix humboldtiana.

Geographical location of the study area $\left(32^{\circ} 03^{\prime} 43^{\prime \prime} \mathrm{S}\right.$ and $\left.60^{\circ} 38^{\prime} 39^{\prime \prime} \mathrm{W}\right)$. The proximity of the study area to the Parana River (Argentina) stands out. Study area and forest location are delimited as: T: Tessaria integrifolia; A: Albizia inundata; L: Nectandra angustifolia and S: Salix humboldtiana.

Neiff 1990, Zamboni y Aceñolaza 2004), asociado a una mayor evapotranspiración que precipitación.

El área de estudio corresponde a un sector de islas que presentan una morfología propia, con los bordes externos (albardones marginales) más elevados y bajos inundables en su zona interna, con áreas intermedias de transición (media loma). En los albardones marginales se observan bosques monoespecíficos de SSh y STi, albardones internos (con bosques de MAi) y, entre éstos, otros albardones más antiguos con bosques de MNa. Este gradiente topográfico se corresponde con gradientes texturales en el sustrato sedimentario o edáfico, encontrándose las texturas gruesas asociadas a las zonas más altas, influenciadas por los pulsos de inundación (Passeggi 2000, Aceñolaza et al. 2005, 2006, 2008). La activa dinámica de este pulso hidrosedimentológico determina una baja a inexistente pedogénesis, tratándose por lo tanto de suelos poco evolucionados (Entisoles, Pereyra et al. 2004), Arenosoles en los albardones marginales y Fluvisoles (o Regosoles) en los internos (FAO 1994).

Los albardones externos están dominados por bosques monoespecíficos de especies colonizadoras de bancos de arena en el cauce principal (bosques de $T$. integrifolia y de $S$. humboldtiana; Aceñolaza et al. 2005, Oakley et al. 2005). Estructural y florísticamente estos bosques se caracterizan como bosques altos y cerrados, organizados en un estrato arbóreo de $12 \mathrm{~m}$ de altura, un estrato arbustivo de 1,5 $\mathrm{m}$ de altura (constituido principalmente por Hyptis mutabilis (Rich.) Briq.) más un estrato herbáceo de $30 \mathrm{~cm}$ de altura representado por un denso pastizal de Paspalum notatum Alain ex Flüggé y Cynodon dactylon (L.) Pers. (Marchetti y Aceñolaza 2005).

Los bosques estudiados se consideran de riqueza intermedia, aunque se pueden encontrar algunos de baja diversidad en áreas de reciente colonización, ya que la riqueza y diversidad se van acentuando con su madurez. Los estratos arbustivo y herbáceo son ricos en especies, encontrando Hyptis mutabilis, Baccharis salicifolia (Ruiz et Pav.) Pers. y Lippia alba (Mill.) N.E. Br. entre las arbustivas más frecuentes, y $P$. notatum, $C$. dactylon, Panicum dichotomiflorum Michx. entre las herbáceas más frecuentes.

Los bosques de albardones internos están dominados por MAi (A. inundata), ingá (Inga verna Willd.) o $\mathrm{MNa}$ $(N$. angustifolia) constituyendo, en algunos casos, bosques mixtos. Estos bosques mixtos presentan mayor riqueza específica que los monoespecíficos; poseen un estrato arbóreo superior, de hasta $12 \mathrm{~m}$ de altura dominado por MAi y MNa; puede encontrarse un estrato arbóreo inferior a $6 \mathrm{~m}$ de altura con sangre de drago (Croton urucurana Baill.); uno arbustivo, de 1,5 m, dominado por Teucrium vesicarium Mill., más otro herbáceo de $50 \mathrm{~cm}$ de altura, con $P$. sabulorum Lam. y Solanum reflexum Schrank, y especies volubles como Melothria candolleana Cogn., Mikania cordifolia (L. f.) Willd., Ipomoea cairica (L.) Sweet y Vigna adenantha (G. Mey.) Maréchal, Mascherpa et Stainier (Aceñolaza et al. 2005).

En cuanto a las características fenológicas de las especies estudiadas, el SSh y el STi se consideran semicaducifolias (Jozami y Muñoz 1984) y colonizadoras (Aceñolaza et al. 2005).

La densidad de arbolado es muy variable según la etapa sucesional considerada. En el caso de los bosques estudiados se encontraron las siguientes características: a) bosques monoespecíficos de SSh y STi con aproximadamente 820 árboles $\mathrm{ha}^{-1} ;$ b) el MAi es una especie caducifolia (Jozami y Muñoz 1984), siendo la densidad de arbolado próxima a los 1.060 árboles ha ${ }^{-1}$; y c) el $\mathrm{MNa}$ es la única especie perennifolia (Jozami y Muñoz 1984), siendo su densidad arbórea más baja y su distribución espacial regular (100 árboles $h^{-1}$ ).

Metodología. Se analizaron durante un período de cinco años (1998 a 2002) los citados cuatro bosques representativos de la llanura aluvial río Paraná en el área del predelta (Argentina) siguiendo la metodología propuesta por Gauch (1982). Los datos no son continuos para todo el período debido a una interrupción involuntaria en el muestreo para el año 1999.

Se analizaron (por bosque) la composición de la hojarasca por fracciones, el aporte medio anual de hojarasca, la variación interanual y la evolución mensual del aporte para el período de estudio. Los valores ofrecidos 
en este trabajo corresponden a la caída de hojas y otras estructuras vegetativas (ramas) o reproductivas (flores) que periódicamente caen de los árboles y conforman lo que se denomina hojarasca. Para estimar la caída de hojarasca se siguió el método del aporte de residuos vegetales al suelo (Aceñolaza et al. 2006). Se recolectó mensualmente el material vegetal caído en trampas de $0,25 \mathrm{~m}^{2}$ por bosque (cuatro trampas por bosque en los bosques monoespecíficos y en el de MAi, y tres en el de MNa). El material se secó (a $70^{\circ} \mathrm{C}$ hasta alcanzar peso constante); posteriormente se pesó y clasificó en cuatro categorías; a) hojas, b) ramas, c) flores+frutos (no se diferenciaron unos de otros debido a su pequeño tamaño) de la especie dominante, y d) otros, donde se incluyó el material vegetal de las demás especies (ya fueran arbóreas, arbustivas o herbáceas) que integran el bosque, además de incluirse otros fragmentos no reconocibles. Los resultados, en todo caso, se expresaron en $\mathrm{Mg}$ de materia seca (MS) ha-1 año-1.

Cabe mencionar que para el bosque de MNa no se tendrá en cuenta su variación interanual para el análisis estadístico, debido a que sólo se disponen datos de dos años.

Las alturas hidrométricas corresponden a las registradas por la Prefectura Naval Argentina para la localidad de Diamante. Se distinguieron por meses completos, de acuerdo con el hemisferio austral, los siguientes períodos: invierno (julio a septiembre), primavera (octubre a diciembre), verano (enero a marzo) y otoño (abril a junio).

No se dispuso de mediciones del contenido de humedad del suelo, pero es lógico suponer un gradiente de mayor a menor humedad de acuerdo a la distancia a los cursos de agua; consecuentemente los bosques marginales presentarán mayor humedad en períodos de inundaciones.

Análisis estadístico. Se aplicaron pruebas de normalidad (Shapiro-Willks modificado) para analizar la distribución del aporte de hojarasca de los cuatro bosques. A pesar de la realización de transformaciones de datos, éstos no se ajustaron a una distribución normal, por lo que se aplicó la prueba de Kruskal-Wallis (KW) que permite realizar un análisis de varianza no paramétrico (estadístico $\mathrm{H}$ ), siendo la variable dependiente el aporte de hojarasca y los criterios de clasificación los bosques, las fracciones y las fechas.

El grado de asociación entre las variables de aporte de hojarasca por bosque y nivel hidrométrico se obtuvo a partir del coeficiente de correlación de Spearman (medida no paramétrica de asociación basada en rangos, que puede usar variables no necesariamente normales).

Se correlacionó el aporte total de cada bosque y el aporte de las hojas con la altura del río para los años (1998 y 2002) y meses (marzo, abril y mayo), significativamente diferentes.

Estas técnicas de muestreo, tratamiento y análisis de datos (InfoStat 2002) fueron profusamente ensayadas en otros estudios (Neiff y Poi de Neiff 1990, Santa Regina et al. 1991, Clarke y Allaway 1996, Williams-Linera y Tolomé
1996, Grigg y Mulligan 1999, Haase 1999, Carnevale y Lewis 2001, Zamboni y Aceñolaza 2004).

\section{RESULTADOS}

Producción media anual de hojarasca. La caída media anual (1998-2002) de hojarasca de los bosques estudiados varió de 3,3 en MAi (el menos productivo) a 9,6 $\mathrm{Mg} \mathrm{MS} \mathrm{ha}^{-1}$ $\mathrm{año}^{-1}$ en $\mathrm{MNa}$ (el más productivo); esto es, los bosques mixtos se colocaron en los extremos de la producción de hojarasca (cuadro 1). Por tanto, los dos bosques mixtos presentaron diferencias significativas tanto entre sí como con los bosques monoespecíficos $(\mathrm{H}=243 ; P<0,001)$, no encontrándose diferencias significativas entre las producciones de los dos bosques monoespecíficos $(P>0,01)$.

En lo que respecta a la composición por fracciones en los bosques de STi, MNa y MAi las hojas contribuyeron con menos del $40 \%$ del total de la hojarasca, mientras que en el bosque de SSh las hojas conformaron el 69\% (cuadro 1). El aporte de las hojas difirió significativamente en los cuatro bosques estudiados $(\mathrm{H}=336 ; P<0,001)$. Las ramas constituyeron del 6 al 18\% de la hojarasca (cuadro $1)$; el mayor aporte de ramas correspondió al bosque de SSh (1,2 Mg MS ha-1 año $\left.{ }^{-1}\right)$ que fue significativamente diferente a los demás $(\mathrm{H}=87,1 ; P<0,001)$. La contribución de las estructuras reproductivas fue baja (menos del $3 \%$ ) (cuadro 1). Los bosques mixtos presentaron valores significativamente más bajos de flores y frutos que los monoespecíficos (colonizadores). A su vez, estos dos últimos bosques también difirieron significativamente entre sí $(\mathrm{H}=204 ; P<0,001)$.

Más del $50 \%$ del aporte total de los bosques de STi, MNa y MAi deriva de otras especies que conforman cada bosque. En el bosque monoespecífico de STi este aporte proviene de hojas de SSh, tanto de los árboles que se encuentran dentro del bosque de STi, como probablemente de hojas transportadas por el viento. Consecuentemente, el aporte de "otros" (cuadro 1) fue significativamente diferente en los cuatro bosques $(\mathrm{H}=347 ; P<0,001)$, obteniéndose el mayor valor en el bosque de $\mathrm{MNa}(5,30$ Mg MS ha ${ }^{-1}$ año ${ }^{-1}$, suponiendo el $55 \%$ de la caída total aérea) y el menor en el de SSh $\left(0,80 \mathrm{Mg} \mathrm{MS} \mathrm{ha-1} \mathrm{año}^{-1}\right.$, representando $10 \%$ de la producción total de la hojarasca), siendo el único bosque donde el aporte de hojas propias alcanzó $69 \%$ del total de la producción.

Variación interanual de la caída de hojarasca. Considerando la producción total interanual de hojarasca de los bosques analizados, el aporte durante el año 2002 fue significativamente inferior que el observado para el resto de los años en los bosques de STi, SSh y MAi (STi: H = 12,9; $P=0,005$. SSh: $\mathrm{H}=13,9 ; P<0,005$. MAi: $\mathrm{H}=8,96$; $P<0,05)$.

El aporte de las otras especies presentes en el bosque disminuyó significativamente en el bosque monoespecífico 
Cuadro 1. Caída anual de hojarasca (en $\mathrm{Mg} \mathrm{MS} \mathrm{ha}^{-1} \mathrm{año}^{-1}, \pm$ intervalo de confianza con $\alpha=0,5$ ) de bosques simples de $T$. integrifolia (STi) y S. humboldtiana (SSh) y mixtos dominados por A. inundata (MAi) y N. angustifolia (MNa) ubicados en el PNPD (período 1998-2002).

Annual litterfall (in Mg (of dry matter) DM/ha/year, \pm confidence interval with $\alpha=0.5$ ) for simple forests of $T$. integrifolia (STi) and S. humboldtiana (SSh), and mixed forest dominated by A. inundata (MAi) and N. angustifolia (MNa) located in the PNPD (Period 1998-2002).

\begin{tabular}{|c|c|c|c|c|c|c|c|c|}
\hline Bosque & $\begin{array}{l}\text { Número } \\
\text { muestras }\end{array}$ & Año & Hojas & Ramas & $\begin{array}{l}\text { Flores } \\
\text { +frutos }\end{array}$ & Otros & Total & Hojas/ramas \\
\hline \multirow{6}{*}{$\begin{array}{l}\text { Salix } \\
\text { humboldtiana } \\
\text { SSh }\end{array}$} & 48 & 1998 & $5,58 \pm 0,15$ & $1,4 \pm 0,10$ & $0,25 \pm 0,01$ & $0,97 \pm 0,05$ & $8,2 \pm 0,16$ & 4,0 \\
\hline & 48 & 2000 & $4,83 \pm 0,16$ & $1,1 \pm 0,06$ & $0,19 \pm 0,01$ & $0,63 \pm 0,03$ & $6,9 \pm 0,18$ & 4,0 \\
\hline & 48 & 2001 & $5,04 \pm 0,13$ & $1,1 \pm 0,07$ & $0,12 \pm 0,01$ & $0,67 \pm 0,04$ & $6,9 \pm 0,16$ & 4,8 \\
\hline & 48 & 2002 & $3,15 \pm 0,14$ & $1,2 \pm 0,09$ & $0,13 \pm 0,01$ & $0,77 \pm 0,05$ & $5,3 \pm 0,21$ & 2,5 \\
\hline & 192 & Media & $4,7 \pm 1,0$ & $1,2 \pm 0,2$ & $0,2 \pm 0,1$ & $0,8 \pm 0,2$ & $6,8 \pm 1,2$ & 3,9 \\
\hline & & $\%$ & 69 & 18 & 3 & 10 & 100 & - \\
\hline \multirow{6}{*}{$\begin{array}{l}\text { Tessaria } \\
\text { integrifolia STi }\end{array}$} & 48 & 1998 & $2,41 \pm 0,08$ & $0,8 \pm 0,04$ & $0,24 \pm 0,04$ & $4,35 \pm 0,11$ & $7,8 \pm 0,20$ & 3,2 \\
\hline & 48 & 2000 & $2,03 \pm 0,06$ & $0,8 \pm 0,06$ & $0,11 \pm 0,01$ & $3,68 \pm 0,08$ & $6,6 \pm 0,16$ & 2,5 \\
\hline & 48 & 2001 & $2,44 \pm 0,08$ & $0,6 \pm 0,03$ & $0,14 \pm 0,02$ & $3,72 \pm 0,07$ & $6,9 \pm 0,10$ & 4,1 \\
\hline & 48 & 2002 & $1,64 \pm 0,09$ & $0,6 \pm 0,04$ & $0,10 \pm 0,01$ & $2,32 \pm 0,08$ & $4,6 \pm 0,20$ & 2,7 \\
\hline & 192 & Media & $2,1 \pm 0,4$ & $0,7 \pm 0,1$ & $0,1 \pm 0,1$ & $3,5 \pm 0,9$ & $6,5 \pm 1,3$ & 3,0 \\
\hline & & $\%$ & 33 & 10 & 2 & 55 & 100 & - \\
\hline \multirow{6}{*}{$\begin{array}{l}\text { Albizia inundata } \\
\text { MAi }\end{array}$} & 44 & 1998 & $0,87 \pm 0,07$ & $0,6 \pm 0,06$ & $0,04 \pm 0,01$ & $1,5 \pm 0,08$ & $3,0 \pm 0,10$ & 1,2 \\
\hline & 48 & 2000 & $1,23 \pm 0,10$ & $0,1 \pm 0,02$ & $0,03 \pm 0,01$ & $2,42 \pm 0,09$ & $3,8 \pm 0,12$ & 11,1 \\
\hline & 48 & 2001 & $1,19 \pm 0,14$ & $0,4 \pm 0,04$ & $0,05 \pm 0,01$ & $1,79 \pm 0,06$ & $3,4 \pm 0,14$ & 3,3 \\
\hline & 47 & 2002 & $0,59 \pm 0,03$ & $0,5 \pm 0,03$ & $0,03 \pm 0,01$ & $1,52 \pm 0,09$ & $2,6 \pm 0,08$ & 1,3 \\
\hline & 185 & Media & $1,0 \pm 0,3$ & $0,4 \pm 0,2$ & $0,04 \pm 0,01$ & $1,8 \pm 0,4$ & $3,3 \pm 0,5$ & 2,5 \\
\hline & & $\%$ & 30 & 13 & 1 & 57 & 100 & - \\
\hline \multirow{6}{*}{$\begin{array}{l}\text { Nectandra } \\
\text { angustifolia } \mathrm{MNa}\end{array}$} & 36 & 1998 & $4,73 \pm 0,17$ & $0,5 \pm 0,02$ & $0,07 \pm 0,01$ & $5,06 \pm 0,33$ & $10,3 \pm 0,33$ & 10,1 \\
\hline & 36 & 2000 & $2,65 \pm 0,18$ & $0,6 \pm 0,05$ & $0,01 \pm 0,00$ & $5,51 \pm 0,14$ & $8,8 \pm 0,24$ & 4,3 \\
\hline & 0 & 2001 & nd & nd & nd & nd & nd & nd \\
\hline & 0 & 2002 & nd & nd & nd & nd & nd & nd \\
\hline & 72 & Media & $3,7 \pm 1,5$ & $0,5 \pm 0,1$ & $0,04 \pm 0,01$ & $5,3 \pm 0,3$ & $9,6 \pm 1,1$ & 6,2 \\
\hline & & $\%$ & 39,0 & 6,0 & 0,4 & 54,6 & 100 & - \\
\hline
\end{tabular}

Se indica la proporción de cada fracción (hojas, ramas, flores/frutos) de la especie dominante y de otras especies del bosque ("otros") con la que contribuyen al total del aporte del bosque (\%) y la variación de la relación de caída de hojas/ramas; nd: sin datos. Nótese que los resultados de la relación entre los componentes 'hojas/ramas' (última columna del cuadro) se obtuvieron promediando los valores de cada muestra y, por tanto, no pueden extrapolarse a partir de los promedios presentados para cada uno de estos componentes (columnas 4 y 5 del cuadro).

de STi en 2002 (año de menor caída). En los bosques de MAi y SSh no se observó una disminución interanual, si bien el aporte de otras especies fue significativamente mayor en el año 2000 para MAi, alcanzando un valor de 2,42 Mg MS ha-1 año ${ }^{-1}$, mientras que en SSh el año de menor aporte fue el 2000 con un valor de $0,63 \mathrm{Mg} \mathrm{MS}$ ha $^{-1}$ año $^{-1}$ (cuadro 1; SSh: $\mathrm{H}=12,3, P=0,006$. STi: $\mathrm{H}=44,7, P<0,001$. MAi: $\mathrm{H}=18,8 ; P<0,005)$.

Se observó una disminución interanual de caída de hojas en los cuatro bosques desde 1998 a 2002, siendo el máximo aporte foliar en 1998 para SSh. En el bosque de MAi no se observaron diferencias significativas entre años, es decir, no habría afectado la variación del nivel del río (cuadro 1), mientras que para los bosques monoespecíficos las diferencias sí son estadísticamente significativas (SSh: $\mathrm{H}=42,5 ; P<0,001$. STi: $\mathrm{H}=18,6 ; P<0,005$. MAi: $\mathrm{H}=2,8 ; P>0,05)$.

No se observaron variaciones significativas en el aporte de ramas desde 1998 a 2002 (cuadro 1) salvo para el MAI que, en el año 2000 presentó valores significativamente más bajos (MAi: $\mathrm{H}=31,2 ; P<0,001$. STi: $\mathrm{H}=4,44$; $P>0,05$. SSh: $\mathrm{H}=0,64 ; P>0,05)$. Tampoco se observaron variaciones interanuales en el aporte de estructuras reproductivas en los bosques de STi y MAi. En el bosque de SSh el aporte de flores y frutos fue significativamente mayor en 1998 (cuadro 1; STi: $\mathrm{H}=4,6 ; P>0,05$. MAi: $\mathrm{H}=0,54 ; P>0,05$. SSh: $\mathrm{H}=22,4 P<0,001$. No se incluyeron los resultados de $\mathrm{MNa}$ ). 
Cuadro 2. Valores medios mensuales ( \pm error estándar) de aportes de hojarasca $\left(\mathrm{Mg} \mathrm{MS} \mathrm{ha}^{-1}\right.$ año $\left.^{-1}\right)$ de bosques monoespecíficos de T. integrifolia (STi) y S. humboldtiana (SSh), y mixtos de A. inundata (MAi) y N. angustifolia (MNa), ubicados en el PNPD, durante cada año de estudio (1998, 2000, 2001 y 2002)*. Se destaca el aporte medio mensual de cada bosque (media total) para el total del período 1998-2002; nd: sin datos.

Monthly values ( \pm standard error) of litterfall (Mg DM/ha/year) for simple forests of T. integrifolia (STi) and S. humboldtiana (SSh); and mixed forest dominated by A. inundata (MAi) and N. angustifolia (MNa) located in the PNPD, during the study period (1998, 2000, 2001 and 2002)*. The monthly contribution of each forest (average) for the total of period 1998-2002, stands out. nd: no data available.

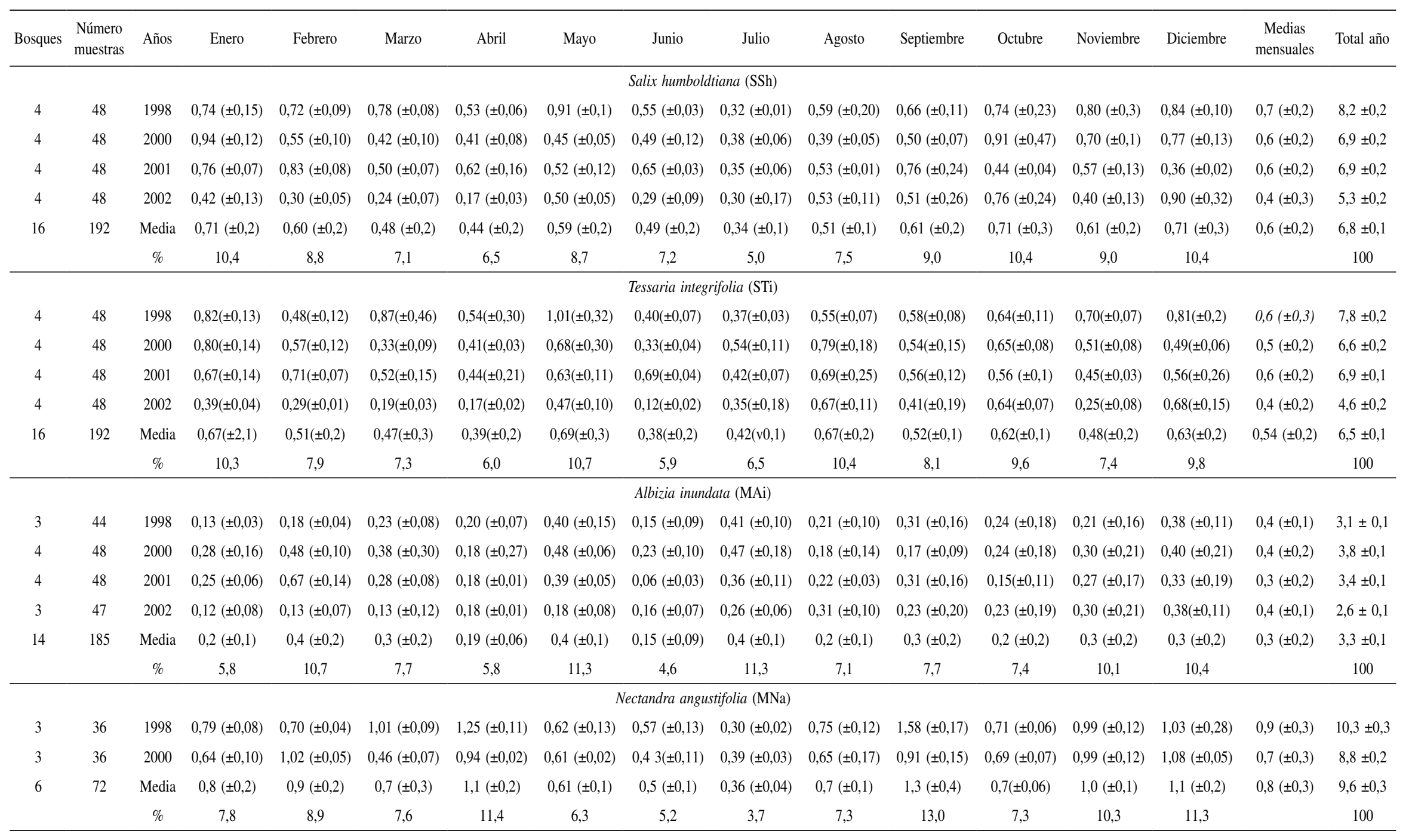

* En el bosque de Nectandra angustifolia (MNa) no se hicieron registros durante los años 2001 y 2002. 
Evolución mensual del aporte. El patrón de aporte de hojarasca en los cuatro bosques estudiados es primaveralestival (principalmente de noviembre a febrero). Pero al intentar discriminar los aportes por estaciones durante el período de estudio no se encontraron diferencias significativas, salvo para el bosque monoespecífico de SSh ( $\mathrm{H}=19,7 ; P<0,001)$.

Sin embargo, si se considera el aporte mes a mes, las diferencias se vuelven significativas también en los bosques de STi y MAi, resultando los mayores aportes los meses de primavera-verano (principalmente de noviembre a febrero, con cerca del $40 \%$ del aporte anual en estos meses). En estos bosques caducifolios y semicaducifolios suele haber un pico secundario de aporte en el período otoñal (mayo a junio), registrándose más del 10\% del aporte de la hojarasca anual en el mes de mayo, a diferencia de la especie perennifolia $\mathrm{MNa}$, ya que no se observaron diferencias entre meses (STi: $\mathrm{H}=51,0, P<0,001$. MAi: $\mathrm{H}=42,3$; $P<0,001$. MNa: $\mathrm{H}=9,35 ; P>0,05)$.

La caída de hojas fue significativamente mayor en los meses de primavera-verano en los bosques monoespecíficos (STi y SSh), mientras que en los bosques mixtos (MNa y MAi) se destacó un pico de aporte en los meses de otoño, permitiendo identificar un patrón de caída en verano-otoño, ya que aproximadamente un $50 \%$ de las hojas cayó en ese período (figura 2; SSh: $\mathrm{H}=103 ; P<0,001$. STi: $\mathrm{H}=35,4 ; P<0,001$. MAI: $\mathrm{H}=20,5 ; P<0,001$. MNa: $\mathrm{H}=11,2 ; P<0,05)$.

La caída de ramas mostró un patrón menos claro, pero se observó que, generalmente, fue más marcada a partir de agosto. Se observaron diferencias significativas en el bosque de SSh para los meses finales de invierno y primavera. En el bosque de MAi la caída de ramas fue significativamente menor en los meses de primavera-verano. No se observaron diferencias significativas para STi, a pesar que el $50 \%$ de las ramas cayó de agosto a noviembre; tampoco para $\mathrm{MNa}$, a pesar que en los meses de mayo y octubre las ramas llegaron a representar más del $10 \%$ de la hojarasca total recolectada (figura 2; SSh: $\mathrm{H}=14,4 ; P<0,05$. MAi: $\mathrm{H}=7,93 ; P<0,05$. STi: $\mathrm{H}=3,21 ; P>0,05$. MNa: $\mathrm{H}=4,52 ; P>0,05)$.

Se identificó un patrón estacional de caída de flores y frutos muy marcado para los bosques mixtos; así, en el bosque de MAi en octubre (primavera) cayó el $70 \%$ de flores y frutos, y en el de MNa entre marzo y abril (fines de verano-otoño) se contabilizó el $60 \%$. Además, en el bosque de MAi las estructuras reproductivas representaron ese mes de octubre más del $15 \%$ de la hojarasca recolectada, lo que es destacable dado su pequeño tamaño y peso. Por tanto, hubo diferencias altamente significativas con el resto de los meses (MAi: $\mathrm{H}=20,2 ; P<0,001$. MNa: $\mathrm{H}=9,64$; $P<0,01)$. En los bosques monoespecíficos, las flores y frutos cayeron a lo largo del año. Sin embargo, en el bosque de STi se concentraron significativamente en los meses de otoño-invierno $(\mathrm{H}=41,3 ; P<0,001)$, siendo abril el de mayor aporte (36\% del total de flores del año). En el bosque de SSh se observaron diferencias significativas sólo entre algunos años (2000 y 2001; $\mathrm{H}=41,9 ; P<0,001)$ donde los meses de primavera-verano presentaron valores significativamente altos; el resto de los años no mostró diferencias significativas $(H=4,09 ; P>0,05)$.

En lo referente al aporte mensual de otras especies que conforman el bosque entre agosto y octubre se concentró más del 70\% del aporte en el SSh, presentando valores significativamente mayores que en los demás; en el de STi no se observaron diferencias significativas. En cuanto a los bosques mixtos de MAi y $\mathrm{MNa}$, cerca del $50 \%$ del aporte de las otras especies se registró en primavera (entre los meses de septiembre y diciembre) mientras que para el de MAi se encontraron diferencias significativas no fue así para el de MNa (MAi: $\mathrm{H}=24,7 ; P<0,001$. MNa: $\mathrm{H}=2,95 ; P>0,05$. SSh: $\mathrm{H}=30,5, P<0,001$. STi: $\mathrm{H}=2,87 ; P>0,05)$.

Relación entre la altura hidrométrica y el aporte de hojarasca. La altura del río (figura 2) fue significativamente más alta en el año 1998 y muy baja en 2002, pudiendo así interpretarse que este último año fue seco $(\mathrm{H}=96,42$; $P<0,001)$. Por otra parte, la altura hidrométrica $\left(h_{\mathrm{r}}\right)$ del río mostró un incremento significativo durante los meses de marzo, abril y mayo respecto a los meses de primavera y verano, indicando un claro pulso de inundación otoñal, mientras que el nivel del río bajó el resto el año, especialmente en enero $(H=183 ; P<0,001)$.

Al relacionar el aporte total de cada bosque (y el de hojas) con la altura del río para los años (1998 y 2002) y meses (marzo, abril y mayo), significativamente diferentes, se aprecia que en las especies semicaducifolias y caducifolias (STi, SSh y MAi) la variación hidrométrica condicionó la caída de hojarasca del bosque (SSh: $r=0,68 ; P<0,01$. STi: $r=0,53 ; P=0,01$. MAi: $r=0,63 ; P<0,01)$ y de las hojas (SSh: $r=0,54 ; P<0,01$. STi: $r=0,45 ; P<0,05$. MAi: $r=-0,25 ; P>0,05)$ de las especies dominantes, aunque para la especie caducifolia (MAi) la caída de hojas sea independiente de la oscilación de la $\mathrm{h}_{\mathrm{r}}$ de la lámina de agua (coherentemente, al ser, como caducifolia, más dependiente de la temperatura ambiental).

Para la especie perennifolia (MNa) el aporte del bosque no presentó relación con $\mathrm{h}_{\mathrm{r}}(\mathrm{MNa}: r=0,15 ; P>0,05)$.

\section{DISCUSIÓN}

Aporte aéreo anual de hojarasca. En los bosques de SSh, STi y $\mathrm{MNa}$ las producciones de hojarasca son algo mayores que la estimación universal de 5,6 Mg MS ha ${ }^{-1}$ año $^{-1}$ (Brinson et al. 1980) para bosques templados-cálidos y se aproximan a los valores aportados por Haase (1999) para bosques subtropicales sujetos a inundaciones estacionales $(6,9 \mathrm{Mg} \mathrm{MS}$ $\mathrm{ha}^{-1} \mathrm{año}^{-1}$ ). También el valor registrado en el bosque de STi (6,5 Mg MS ha ${ }^{-1}$ año $\left.^{-1}\right)$ resultó inferior al encontrado por Neiff y Poi de Neiff (1990) $\left(8,2 \mathrm{Mg} \mathrm{MS} \mathrm{ha}^{-1}\right.$ año-1) $^{-1}$ 

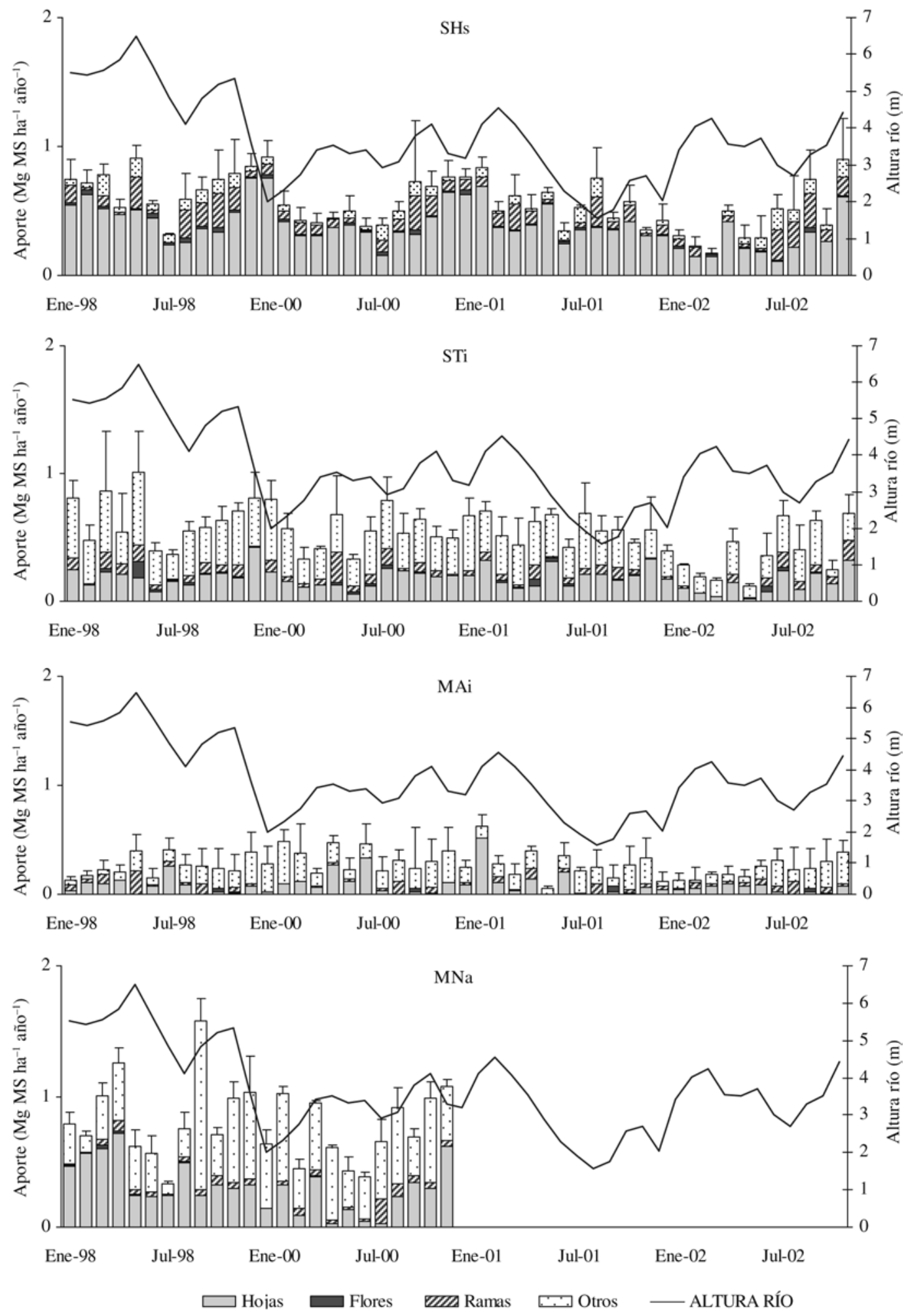

Figura 2. Evolución mensual de aporte de hojarasca (Mg MS ha ${ }^{-1}$ año $\left.^{-1}\right)$ en bosques de: T. integrifolia (STi); S. humboldtiana (SSh); A. inundata (MAi); y N. angustifolia (MNa), ubicados en el PNPD (período 1998-2002). Se detalla el aporte de cada fracción (hojas, ramas, flores+frutos) de la especie dominante y el de las demás especies presentes en el bosque ("otros") que, en conjunto, se incluyen en el aporte total del bosque. Se indica también la altura del río.

Monthly evolution of litterfall contribution (Mg DM/ha/year) in forests of: T. integrifolia (STi); S. humboldtiana (SSh); A. inundata (MAi); and N. angustifolia (MNa), located in the PNPD (period 1998-2002). For the dominant species, we present detailed contribution of each fraction (leaves, branches, flowers+fruits). The rest of the contribution represents all fractions not belonging to the dominant species, and they are included in the total contribution of the forest as "other". The level of the river is also indicated. 
para la misma especie. Posiblemente la densidad arbórea explica las diferencias de las producciones, pues la densidad del bosque de STi del P.N.P.D. fue relativamente baja (820 árboles $\mathrm{ha}^{-1}$, indicando madurez del bosque), mientras que Neiff y Poi de Neiff (1990) estimaron una densidad tan alta como 8.000 árboles ha $^{-1}$, por lo que se trataba de un bosque joven, coherente con los resultados. El bosque mixto de MAi, con especies caducifolias, se encuentra muy lejos de aquellas producciones al tener un período de fotosíntesis más reducido.

Obviamente todos los bosques aquí estudiados superan los valores para bosques subtropicales mesotérmicos del norte de Argentina (0,3 Mg MS ha- año $^{-1}$, según Carnevale y Lewis (2001)), fortaleciendo la hipótesis que postula mayor productividad para bosques sujetos a inundación (Brinson et al. 1980, Junk et al. 1989, Haase 1999) al no estar tan limitados por el agua edáfica durante la época estival.

Los bosques estudiados pueden diferenciarse de acuerdo con la contribución de las hojas al aporte total; así, la relación hojas/ramas $(\mathrm{Hj} / \mathrm{Rm})$ se usa a veces como índice de madurez (Martín et al. 1993). Por lo general, la contribución de hojas suele superar con mucho al de ramas (Gallardo et al. 1989), pero ello sólo sucede ampliamente en el $\mathrm{MNa}(\mathrm{Hj} / \mathrm{Rm}$ medio = 6,2; con sólo dos años), lo que es congruente con la baja densidad arbórea. En los demás bosques, a pesar que la contribución de ramas no fue elevada (inferior al 20\%), el índice medio fue siempre inferior a 4,0 (cuadro 1), siendo el más alto el de SSh. Hay que tener en cuenta que la caída de ramas, que en el área de estudio se produce hacia el mes de agosto, suele ir asociada a un incremento de la intensidad del viento. Además, tras un año ventoso (con abundante caída de ramas) suele suceder un período de escaso aporte (Gallardo et al. 1989), como puede observarse para el MAi en el bienio 1998-2000 (cuadro 1), siendo el bosque menos maduro (coherente con su alta densidad arbórea).

Por tanto, si se considera que diferentes factores externos determinan la caída de ramas (vientos) y la de hojas (humedad edáfica) es lógico que la variación interanual del coeficiente $\mathrm{Hj} / \mathrm{Rm}$ no presente un patrón claro a lo largo del período de estudio, pero se apunta la tendencia en cuanto a que la madurez de los sistemas forestales estudiados sigue el orden: $\mathrm{MAi}<\mathrm{STi}<\mathrm{SSh}<\mathrm{MNa}$.

Resalta la alta contribución de "otros" en la producción de hojarasca. Si bien sería esperable en los bosques mixtos (la alta densidad del arbolado en el MAi no compensa su baja productividad), no se entiende bien que este hecho suceda en el monoespecífico de STi, donde las hojas de SSh se mezclan con las propias del STi, sobrestimando el aporte de hojarasca total del STi.

Patrón de la evolución temporal del aporte de hojarasca. La significativamente mayor y más temprana caída primaveral-estival de hojas en los bosques monoespecíficos (STi y SSh) indica que el aporte de hojarasca coincide con el período de mayor actividad fotosintética, mientras que en los bosques mixtos (MAi y MNa) la significativa mayor caída es otoño-invernal, patrón usual de los bosques caducifolios (como el MAi).

El bosque monoespecífico de $\mathrm{SSh}$ es el único donde el aporte de la especie dominante refleja la curva de aporte total del bosque (figura 2). En el bosque de STi, a pesar de considerarse maduro y simple, presenta un patrón de aporte compuesto en su mayoría por hojarasca de SSh, notándose una diferencia significativa al comparar los valores del bosque con los de la especie dominante. Tampoco en los bosques mixtos la contribución de la especie dominante describe el patrón de aporte total del bosque, denotando una baja influencia de la especie dominante en este último.

Relación aporte de hojarasca-nivel hidrométrico. En el período de estudio esta relación varió entre años, disminuyendo desde 1998 (pico de creciente) hasta 2002 (año seco). En estos años se identificaron fases de inundación anual, representados por los meses de marzo, abril y mayo. Se evidenció una relación significativa y positiva entre la $h_{r}$ y el aporte de hojarasca de los bosques semicaducifolios y caducifolios para estos períodos. Estos valores para el bosque perennifolio ( $\mathrm{MNa}$ ) se mostraron independientes de las fluctuaciones de la $h_{r}$.

Por otro lado, en los bosques monoespecíficos semicaducifolios (STi y SSh) el aporte de hojas también estuvo relacionado positivamente con la $h_{r}$, siendo mayor el aporte de nutrientes al suelo en primavera-verano, cuando el pulso de inundación disminuye y la temperatura aumenta, generando un déficit hídrico temporal. Por otro lado, en el bosque caducifolio (MAi) no se estableció tal relación.

En general, se observó que en los bosques monoespecíficos ( $\mathrm{STi}$ y $\mathrm{SSh}$ ), más asociados a la dinámica hidrológica, los picos de caída de hojarasca se producen posteriormente a la fase de inundación (al inicio del estiaje), cuando comienza a bajar el nivel del agua (inicio del verano). En el caso del bosque monoespecífico de SSh hay un evidente patrón tardo-primaveral y la caída de hojarasca coincide con el fin de la fase de inundación (Neiff y Poi de Neiff 1990, Zamboni y Aceñolaza 2004). Estos resultados coinciden con los reportados por Bonomelli et al. (2002) de contribución de hojas en plantaciones de Eucalyptus spp. L'Herit y con los reportados por Huber et al. (1986) para Pinus radiata D. Don en diferentes regiones de Chile. Hay en estos bosques, por tanto, una reacción fenológica del árbol al pulso de inundación, que debe tener profundas repercusiones en dinámica de los ciclos biogeoquímicos y, por tanto, en la cesión de nutrientes al subsistema edáfico (Gallardo et al. 1998). 


\section{CONCLUSIONES}

Las conclusiones deducidas son:

- Se encontró que las producciones áreas de los bosques perennifolios y semicaducifolios del Parque Nacional Pre Delta son del mismo orden que las de bosques subtropicales estacionalmente inundables.

- La disminución de los aportes anuales de hojarasca durante el período de estudio (1998-2002) puede asociarse tanto a una respuesta de la vegetación a la excepcional inundación registrada en 1998, como a la sequía del año 2002. Por tanto, la altura del río condiciona la caída de hojarasca en estos bosques semicaducifolios.

- El bosque caducifolio de Albizia inundata (MAi) es el menos productivo, no estando el aporte de hojarasca condicionado por los pulsos fluviales y recibiendo los aportes de otras especies mayormente en primavera.

- La caída de hojarasca en el bosque perenne de Nectandra angustifolia (MNa) es independiente de las fluctuaciones de la altura del río, presentando características de madurez.

- Las estructuras reproductivas representan un porcentaje bajo en el aporte total anual de hojarasca, observándose mayor caída en las especies colonizadoras (SSh y STi).

- Sólo el bosque monoespecífico de Salix humboldtiana (SSh) mantiene un neto patrón definido y repetitivo de caída tardo-primaveral (tras la fase de inundación, asociado al inicio del déficit hídrico), dado que su contribución foliar es alta y es minoritario el aporte de otras especies durante el invierno.

\section{AGRADECIMIENTOS}

Se agradece a R. D'Angelo, R. Zanello y O. Bejarano la ayuda en la obtención de datos de campo y a la Administración de Parques Nacionales el permiso de realización del estudio en un área natural protegida. Este trabajo fue parcialmente financiado por fondos PEI-CONICET 6374, PICT-ANPCyT 11928 y PID-UNER 2089/01. Se agradecen también los aportes realizados por los árbitros de BOSQUE, los cuales permitieron mejorar la exposición del trabajo.

\section{REFERENCIAS}

Aceñolaza P, W Sione, F Kalesnik, MC Serafín. 2005. Determinación de unidades homogéneas de vegetación en el Parque Nacional Pre-Delta (Argentina). Serie Misc. INSUGEO 14: 81-90.

Aceñolaza PG, LP Zamboni, JF Gallardo. 2006. Ciclos biogeoquímicos de bosques de la llanura de inundación del río Paraná (Argentina): Aporte de hojarasca. In Medioambiente en Iberoamérica. Visión desde la Física y la Química en los albores del sXXI, Gallardo Lancho JF ed. Badajoz, España. Sociedad Iberoamericana de Física y Química Ambiental 2: 529-536.
Aceñolaza PG, LP Zamboni, W Sione, F Kalesnik. 2008 Caracterización de la región superior del Complejo Litoral del Río Paraná: Grandes unidades de ambiente. Serie Misc. INSUGEO 17: 293-308.

Bonomelli C, I Peña, D Suárez. 2002. Contribución de las hojas en el proceso de reciclaje de $\mathrm{N}$ y $\mathrm{P}$ en Eucalyptus $s p$. Bosque 23(1): 61-77.

Bray J, E Gorham. 1964. Litter production in forests of the world. Adv. Ecol. Res. 2: 101-157.

Brinson MM, HD Bradshaw, RN Holmes, J Elkins. 1980. Litterfall, stemflow and throughfall nutrient fluxes in an alluvial swamp forest. Ecology 61: 827-835.

Carnevale N, JP Lewis. 2001. Litterfall and organic matter decomposition in a seasonal forest of the eastern Chaco (Argentina). Rev Biol. Trop. 49 (1): 203-212.

Clarke PJ, WG Allaway. 1996. Litterfall in Casuarina glauca Coastal Wetland Forest. Aust. J. Bot. 44: 373-380.

FAO (Organización de las Naciones Unidas para la Agricultura y la Alimentación, IT). 1994. World Reference Base for Soil Resources. Roma, Italia. FAO. 162 p.

Gallardo JF, I Santa Regina, C San Miguel. 1989. Ciclos biogeoquímicos en bosques de la Sierra de Béjar (Salamanca, España). 1. Producción de hojarasca. Rev. Ecol. Biol. Sol, 26: $35-46$.

Gallardo JF, A Martín, I Santa Regina. 1998. Nutrient cycling in deciduous forest ecosystems of the 'Sierra de Gata' mountains: Nutrient supplies to the soil through both litter and throughfall. Ann. For. Sci. 55: 771-784.

Gauch HG. 1982. Multivariate analysis in community ecology. Cambridge, UK. Univ. Press. 298 p.

Godoy JR, G Petos, J Salo. 1999. Riparian flooded forest of the Orinoco and Amazon basins: a comparative review. Biodiversity \& Conservation 8: 551-586.

Grigg AH, DR Mulligan. 1999. Short note: litterfall from two eucalypt woodlands in central Queensland. Aust. J. Ecol. 24: 662-664.

Haase R. 1999. Litterfall and nutrient return in seasonally flooded and non-flooded forest of the Pantanal, Mato Grosso, Brazil. For. Ecol. and Manage. 117: 129-147.

Huber A, JE Schlatter, C Oyarzún. 1986. Aporte de elementos nutritivos por la hojarasca de un bosque adulto de Pinus radiata. Bosque 7 (2): 59-64.

InfoStat. 2002. Manual InfoStat versión 1.1., Grupo InfoStat, F. C. A. Córdoba, Argentina. Universidad Nacional de Córdoba. 262 p.

Jozami JM, JDD Muñoz. 1984. Árboles y arbustos indígenas de la provincia de Entre Ríos. Santa Fe, Argentina. I.P.N.A.P.S.CONICET-U.N.L. 85 p.

Junk W, P Bayley, R Sparks. 1989. The flood pulse concept in river-floodplain systems. Can. Spec. Publ. Fish. Aquat. Sci. 106: 110-127.

Marchetti Z, P Aceñolaza. 2005 Detección satelital y descripción de patrones de vegetación en islas del Paraná Medio. Serie Misc. INSUGEO 14: 191-197.

Martín A, I Santa Regina, JF Gallardo. 1993. Ciclos biogeoquímicos en un bosque perenne de encina (Quercus rotundifolia) en las proximidades de Salamanca (España): Retorno potencial. In Biogeoquímica de ecosistemas. Valladolid, España. Gallardo JF ed. Junta de Castilla y León. p. 151-160.

Monedero C, V González. 1995. Producción de hojarasca y descomposición en una selva nublada del ramal interior de la Cordillera de la Costa, Venezuela. Ecotropicos 8 (1-2): $1-14$. 
Neiff JJ, A Poi de Neiff. 1990. Litterfall, leaf decomposition and litter colonization of $T$. integrifolia (compositae) in the Paraná River floodplain. Hydrobiology 203: 45-52.

Oakley L, D Prado, J Adamoli. 2005. Aspectos biogeográficos del Corredor fluvial Paraguay-Paraná. Serie Misc. INSUGEO 14: $245-258$.

Passeggi E. 2000. Caracterización Sedimentológica del Material Parental de los Suelos Asociados a los Depósitos de Cauce del Tramo Medio del Río Paraná. Tesis Doctoral. Santa Fe, Argentina. Universidad Católica de Santa Fe. 216 p.

Pereyra FX, V Baumann, V Altinier, J Ferrer, P Tchilinguirian. 2004. Génesis de suelos y evolución del paisaje en el delta del río Paraná. Revista de la Asociación Geológica Argentina 59: 229-242.

Proctor J. 1983. Tropical forest litterfall. I. Problems of data comparison. In Tropical Rain Forest: Ecology and Management. Sutton SL, TC Whitmore, AC Chadwick. Oxford, UK. Blackwell. p. 267-273.

Proctor J. 1984. Tropical forest litterfall. II. The data set. In Chadwick AC, SL Sutton eds. Tropical Rain-Forest: The Leeds Symposium. Proc. Leeds Philosophical \& Literary Soc. Leeds, UK. p. 83-113.
Rojas AE, JH Saluso. 1987. Informe Climático de la Provincia de Entre Ríos. Entre Ríos, Argentina. Publ. Técnica No 14. E.E.A. Paraná. 20 p.

Santa Regina I, JF Gallardo, M Rico, A Martín, HA Gallego, G Moreno, S Cuadrado. 1991. Datos preliminares sobre biomasa aérea, producción y características edafoclimáticas de ecosistemas forestales de Quercus pyrenaica (Sierra de Gata, Salamanca). Stvdia Oec. 8: 147-158.

UNESCO-PNUMA-FAO (Organización de las Naciones Unidas para la Educación, la Ciencia y la Cultura-Programa de las Naciones Unidas para el Medio Ambiente-Organización de las Naciones Unidas para la Agricultura y la Alimentación). 1980. Ecosistemas de los bosques tropicales. Capítulo 10. Producción primaria bruta y neta, parámetros de crecimiento. París, Francia. UNESCO. p. 265-282.

Williams-Linera G, J Tolomé. 1996. Litterfall, temperate and tropical dominant trees, and climate in a mexican lowermontane forest. Biotropica 28: 649-656.

Zamboni P, P Aceñolaza. 2004. Aporte al conocimiento de ciclos de materia orgánica en formaciones boscosas de la llanura de inundación del río Paraná. Serie Misc. INSUGEO 12: $5-12$.

Recibido: 30.01.09

Aceptado: 08.06.09 